\title{
Estimulación eléctrica vagal en el tratamiento de la epilepsia rebelde. Situación actual
}

\author{
G. García-March; M.J. Sánchez-Ledesma* y J. Broseta *
}

Servicio de Neurocirugía, Hospital Clínico Universitario, Valencia. *Cátedra de Neurocirugía, Departamento de Cirugía, Universidad de Salamanca. Salamanca.

\section{Resumen}

La estimulación intermitente vagal es una técnica de neuroestimulación de reciente incorporación en el tratamiento de pacientes con epilepsia refractaria al tratamiento farmacológico convencional. Hasta el momento presente $y$, a pesar de algunos resultados dispares, se ha mostrado como una alternativa eficaz y segura en el tratamiento de estos pacientes. El presente trabajo de revisión pretende acercar la técnica quirúrgica de implantación, comentar las indicaciones y resultados obtenidos hasta la actualidad, y profundizar en los posibles mecanismos de acción de este tipo de estimulación, para finalmente realizar una aproximación crítica a la misma, intentando puntualizar cúales han sido los problemas e inconvenientes que han impedido la utilización generalizada de esta alternativa quirúrgica antiepiléptica.

PALABRAS CLAVE: Epilepsia. Estimulación eléctrica vagal. Seguridad y eficacia. Mecanismos de acción.

Vagus nerve stimulation for the treatment of refractory epilepsy. State of the art

\section{Summary}

The vagus nerve stimulation (VNS) therapy is a new neurostimulation technique used for treating pharmacoresistant epilepsy. It can be considered an effective and safe alternative for the treatment of refractory epilepsy patients. In the present review, we describe the surgical implantation technique, its indications and results achieved until now. We will also summarize the possible mechanisms of action of VNS therapy. Finally, we will comment on the difficulties and inconvenients that did not allow this antiepileptic surgical technique to become more widely used.

Recibido: 1-06-07. Aceptado: 1-05-08
KEY WORDS: Epilepsy. Vagus nerve stimulation. Save and efficacy. Action mechanisms.

\section{Introducción}

La epilepsia es una patología que afecta al $1 \%$ de la población general y que podría ser considerada en términos globales como el segundo trastorno neurológico en cuanto a incidencia y prevalencia ${ }^{60}$. Esta enfermedad tiene importantes repercusiones psicológicas y económicas tanto en el paciente que la sufre como en la sociedad que tiene que sufragar los gastos que la misma supone. Además, estos factores se ven aumentados por los efectos secundarios en la esfera cognitiva, caracterológica y emocional, que con frecuencia complican los tratamientos con fármacos antiepilépticos a largo plazo. Es por ello que se hace imperiosa la necesidad de buscar e investigar nuevas modalidades de tratamiento efectivo no farmacológico.

El final del siglo XX ha supuesto un avance importante en el tratamiento de las epilepsias. Sin embargo, a pesar de la proliferación actual de estudios que han aumentado el conocimiento sobre las causas de la epilepsia y que han posibilitado la introducción de nuevos fármacos antiepilépticos, se puede afirmar que todavía un $30-40 \%$ de los pacientes no tienen un control satisfactorio de sus crisis ${ }^{11.13 .71}$. En función de ello se calcula que en Estados Unidos, los pacientes tratados correctamente sin un control adecuado de las crisis podrían estar entre 150.000 y 300.000. Extrapolando estos datos, en España serían unos 20.000-40.000 pacientes. Alrededor de un $20-40 \%$ de estos enfermos pueden ser susceptibles de tratamiento quirúrgico convencional, así, solo entre 8.000 y 16.000 pacientes podrían considerarse candidatos apropiados para la realización de lobectomías temporales, resecciones corticales focales, callosotomías, hemisferectomías, resecciones subpiales y otros procedimientos ablativos menos frecuentes. Quedan por lo tanto un grupo de pacientes con epilepsia parcial o generalizada, refractaria al tratamiento farmacológico, en los que la cirugía convencional no ofrece garantías suficientes de eficacia y seguridad, pudiendo producir graves secuelas $\mathrm{y}$ efectos secundarios. Es precisamente en estos grupos 


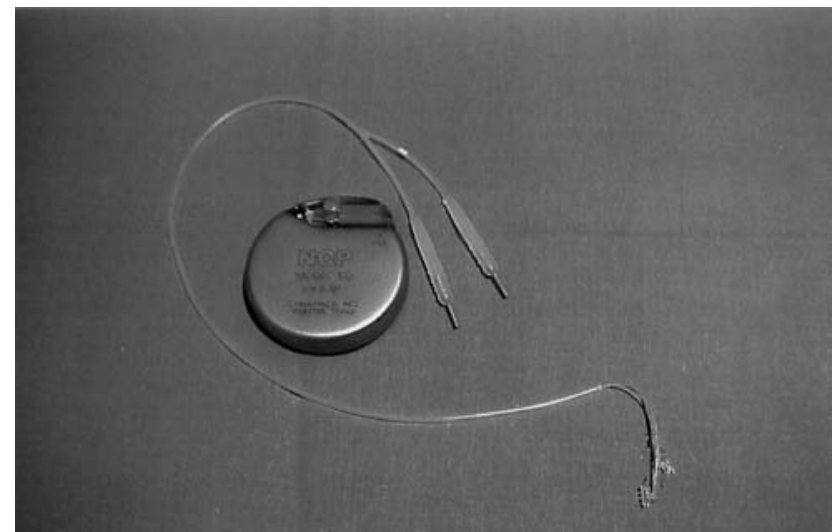

Figura 1. Equipo de estimulación vagal.

donde se están buscando nuevas alternativas terapéuticas y entre ellas, la estimulación eléctrica, de forma crónica, de diferentes estructuras nerviosas ha resultado ser de las más novedosas, seguras y eficaces.

Durante las últimas décadas, gracias al progreso tecnológico y a los avances fisiopatológicos, han adquirido gran auge las técnicas de potenciación de los mecanismos fisiológicos de facilitación o inhibición del sistema nervioso en diferentes campos neuroquirúrgicos. Así, diversas técnicas de neuroestimulación, con resultado dispar, se han venido utilizando en el tratamiento de la epilepsia refractaria al tratamiento farmacológico convencional. La estimulación cerebelosa fue propuesta inicialmente como método de reducción de las crisis epilépticas, fundamentada en la potenciación de las vías inhibitorias de proyección de la corteza cerebelosa. Sin embargo, y después de una serie amplia de ensayos clínicos fue abandonada por ineficaz ${ }^{82}$. La estimulación talámica a nivel del núcleo centromediano ha mostrado hasta el momento resultados dispares en el control de las crisis epilépticas ${ }^{21,77}$. La estimulación crónica del núcleo anterior del tálamo, el locus coeruleus y otras estructuras cerebrales se han propuesto como alternativas en el tratamiento de la epilepsia refractaria con resultados poco concluyentes y repetibles, y aunque para algunos estos estudios preliminares son positivos, se deben considerar con precaución.

Una de las técnicas de neuroestimulación de más reciente incorporación es la estimulación intermitente del nervio vago (VNS). La utilización racional de esta técnica está basada en una serie de hechos experimentales en los que se demuestra la inhibición y bloqueo de las crisis epilépticas focales o generalizadas en diferentes animales de experimentación $\mathrm{n}^{22,44,59.67 .83 .86}$. A partir de estos estudios, y a finales de los ochenta, diversos autores comienzan a utilizar este tipo de estimulación en epilepsias rebeldes refractarias al tratamiento farmacológico, demostrándose en sus resultados preliminares la efectividad de la misma ${ }^{58,63,74}$ Con posterioridad diferentes estudios multicéntricos randomi-

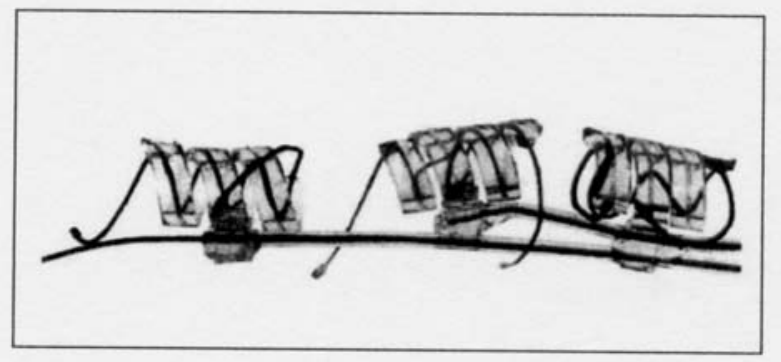

Figura 2. Detalle del terminal helicoidal del electrodo.

zados han confirmado los esperanzadores resultados iniciales $^{6,76}$. Hasta la fecha, y una vez aprobada su utilización como tratamiento alternativo y paliativo por la Food and Drug Administration estadounidense, son ya un número considerable de pacientes a los que se les ha implantado el estimulador vagal.

Sin embargo, a pesar de los buenos resultados recogidos en general con la VNS, existen puntos no evaluados por los diferentes grupos y estudios, uno de ellos es la repercusión en cada uno de los tipos de crisis y síndromes epilépticos. Determinar los factores pronósticos para la respuesta de cada uno de estos tipos es importante porque muchos pacientes epilépticos están limitados en su vida cotidiana por la presencia de un tipo concreto de crisis más que por el número global de las mismas, por su horario, duración, a la asociación de caídas, a que ocurran durante la vigilia o el sueño. Muchas veces el control de esas crisis puede resultar más relevante que la disminución total en el número de episodios críticos. Además, se debe seguir investigando en su mecanismo de acción, por el momento sometido a muchas hipótesis y controversias pero aún desconocido. Igualmente, en el futuro, será necesario perfeccionar los aspectos técnicos del marcapasos, determinar los parámetros óptimos de estimulación, disminuir los efectos secundarios y encontrar nuevas indicaciones. Sólo con la profundización y el estudio de todos estos factores, se podrán determinar los candidatos idóneos para esta moderna técnica.

\section{Descripción del equipo de estimulación. Técnica qui- rúrgica de implantación}

El sistema de estimulación del nervio vago, consta de un generador de impulsos eléctricos de unos $55 \mathrm{~mm}$ de diámetro y $7 \mathrm{~mm}$ de espesor, que se implanta en un bolsillo subcutáneo a nivel infraclavicular, y un electrodo helicoidal bipolar que se dispone alrededor del tramo cervical del nervio vago izquierdo, y que está unido por un cable de conexión con el generador. El procedimiento quirúrgico requiere la realización de dos incisiones diferentes, una a nivel cervical para la implantación del electrodo de estimulación; y, otra a nivel infraclavicular para el alojamiento del generador. 
El generador de impulsos eléctricos es una batería simple de litio encastada en un módulo de titanio, similar al de los marcapasos cardíacos, que en su parte superior tiene una cabeza de resina con un receptáculo para adaptar el cable de conexión que prolonga el electrodo de estimulación (Figura 1).

El electrodo de estimulación está compuesto por dos terminales de platino incrustados en una estructura helicoidal de silicona de tres vueltas, y de otra estructura también helicoidal que ayudará a la fijación y anclaje del electrodo al nervio. Estas estructuras tienen además unos hilos finos que se extienden por el helicoide sobresaliendo por sus extremos, y que permiten la manipulación de los terminales sin dañar los contactos de platino (Figura 2).

El generador se activa e interroga de forma no invasiva a través de un campo magnético inducido telemétricamente por una cabeza programadora que se conecta a un ordenador que integra un software de NCP. El generador produce una corriente alterna de ondas cuadradas que pueden ser programadas de acuerdo a los siguientes parámetros: intensidad, frecuencia, anchura de pulso y tiempos de activación y desactivación de la corriente. La programación de estos parámetros varía según la experiencia de los diferentes centros y en todos los casos deben adecuarse a la tolerancia y efectos de cada paciente. Además de está activación automática programada, el equipo puede activarse pasando un pequeño imán sobre el bolsillo infraclavicular donde se aloja el generador. De esta forma el propio paciente o alguien de su entorno pueden frenar o abortar una crisis que cree inminente por la existencia de algún tipo de aura.

El generador tiene una duración limitada. Utilizando los parámetros de estimulación habituales la vida media de la batería suele ser de unos 5 años, tras los que puede ser reemplazada con anestesia local, de forma ambulatoria y en una intervención que suele durar menos de una hora.

La técnica e instrumental quirúrgico requeridos para la implantación del sistema de estimulación no son extraordinarios, y aunque la intervención puede realizarla cualquier cirujano con experiencia en la exposición del paquete vasculonervioso carotídeo, se recomienda que la realice un neurocirujano, puesto que suelen estar familiarizados en programas y protocolos de epilepsia.

La cirugía suele durar alrededor de dos horas y generalmente se realiza bajo anestesia general. Aunque puede practicarse de forma ambulatoria, es deseable que el paciente tras la intervención, permanezca ingresado para un mejor control de la disfunción de las cuerdas vocales, disfagia, alteraciones respiratorias o crisis epilépticas inducidas por la anestesia. La administración profiláctica de antibióticos es conveniente como paso previo a la intervención, y deben mantenerse unos días después de la misma.

Con el paciente en decúbito supino y el cuello en ligera

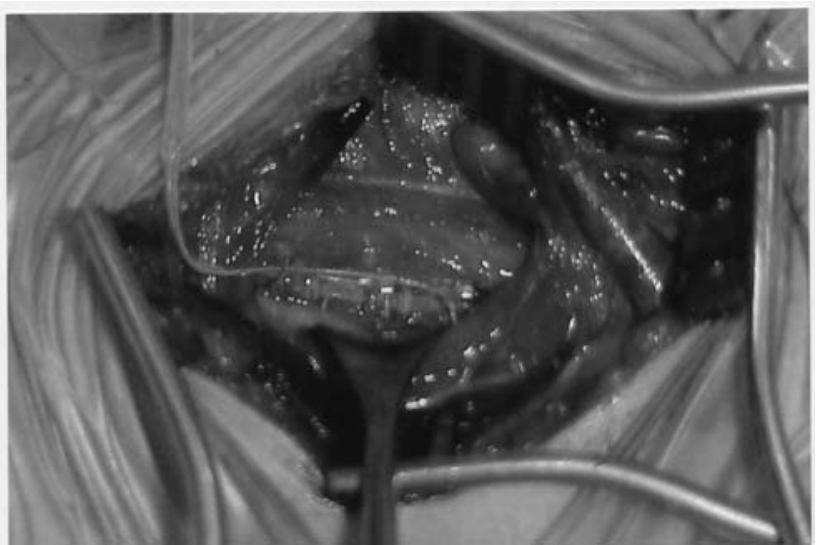

Figura 3. Instantánea quirúrgica del emplazamiento del electrodo alrededor del nervio vago.

extensión, se realiza una primera incisión transversa de unos $7 \mathrm{~cm}$ en el área infraclavicular izquierda a través de la que se diseca un bolsillo subcutáneo sobre la fascia del músculo pectoral, que servirá de alojamiento del generador. En un segundo tiempo, se realiza una nueva incisión transversa de unos 5-6 cm a nivel cervical medio izquierdo, en la que tras dividir el platisma y abrir a lo largo del borde anterior del músculo esternocleidomastoideo la fascia profunda cervical, se identificará el espacio carotídeo cervical. El nervio vago suele encontrarse a nivel del cartílago tiroides, en profundidad y en el lado medial de la vena yugular interna y envuelto en el tejido lateral de la arteria carótida común. Generalmente suele bastar con la disección de unos $3 \mathrm{~cm}$ de longitud del nervio para la colocación correcta del electrodo helicoidal de estimulación. La figura 3 muestra en una instantánea quirúrgica el electrodo emplazado alrededor del nervio vago. Para el paso de la conexión del electrodo entre las dos heridas, se dispone de un tunelizador especial que realiza un trayecto subcutáneo, minimizando las lesiones en las estructuras vasculares cervicales. En el mismo quirófano y antes de cerrar las heridas quirúrgicas se realizan los primeros test electrodiagnósticos del generador y del electrodo para comprobar el buen funcionamiento y conexión entre los mismos y el propio nervio vago. Las figuras 4 y 5 esquematizan la disposición del electrodo y el generador en el paciente.

La estimulación intermitente vagal suele comenzar una semana después de la cirugía, iniciándose con unos parámetros eléctricos, que se programan transcutaneamente con el cabezal telemétrico comentado, de $0.5 \mathrm{~mA}$ de intensidad, $30 \mathrm{~Hz}$ de frecuencia, $500 \mathrm{mseg}$ de amplitud de onda de pulso y manteniendo una intermitencia de 30 seg de estimulación cada 10 minutos durante las 24 horas del día. Con posterioridad, de forma ambulatoria, cada dos semanas y durante los tres primeros meses, se puede modificar el programa de estimulación, atendiendo a la eficacia y tolerancia en cada uno de los pacientes. Una vez determi- 


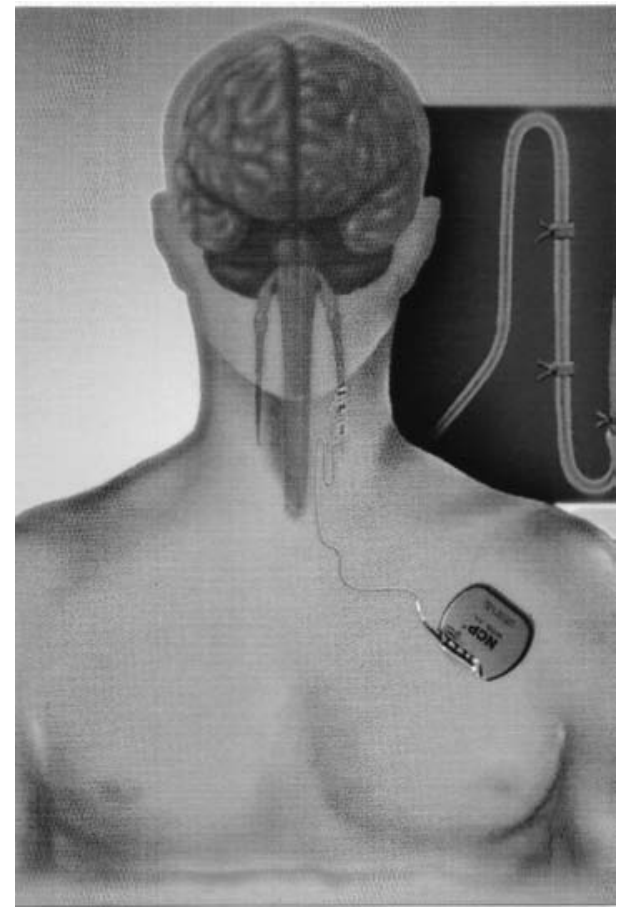

Figura 4. Esquema de la disposición del electrodo y generador en el paciente.

nada la efectividad del sistema se suelen realizar controles periódicos trimestrales.

Los pacientes suelen disponer de un pequeño imán con el que, en caso de necesidad, pueden activar el generador aplicándolo sobre el mismo durante 1 a 3 seg. Si se mantiene apoyado sobre el generador durante más tiempo se produce un bloqueo de la estimulación. Del primer modo el paciente puede frenar o abortar una crisis. Del segundo, se detiene el funcionamiento del estimulador en el momento en que se producen molestias importantes al paciente. Se hace por ello preciso que el paciente, la familia y el cuidador entiendan bien el sistema y las señales de alarma, y así puedan actuar oportunamente en caso de crisis o de efectos secundarios.

Como se ha comentado, el generador tiene una duración limitada. Dependiendo del nivel de la estimulación y de la resistencia del electrodo, puede oscilar entre cuatro y doce años en función del modelo de generador empleado. Pasado este tiempo es necesario proceder al recambio del generador mediante una nueva cirugía, esta vez sin modificar el electrodo. Este último, una vez fijado en la cirugía inicial, queda adherido al nervio vago por una rica fibrosis local. Puede extraerse sin problemas, pero la práctica habitual es mantenerlo en su lugar aunque deje de utilizarse ${ }^{14}$.

Al no interferir con otros tratamientos, la colocación del estimulador vagal prácticamente no tiene contraindicaciones absolutas. En pacientes con arritmias cardíacas, alteraciones autónomas o trastornos de la coagulación, su

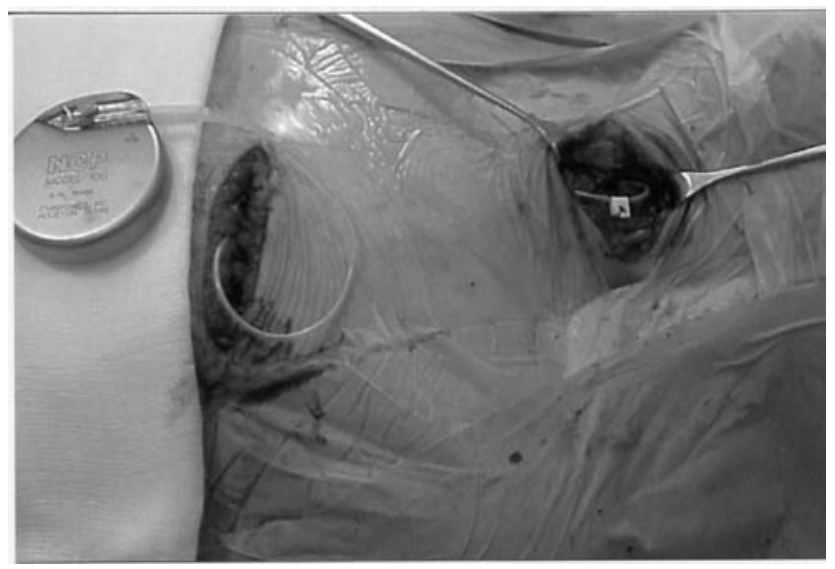

Figura 5. Instantánea quirúrgica del electrodo tunelizado y conectado al generador de impulsos eléctricos, una vez realizada la bolsa subcutánea que lo alojará definitivamente.

indicación debe meditarse bien por los riesgos que puede conllevar la cirugía de colocación o la propia estimulación. Recientemente, los diseñadores han notificado la contraindicación de tratamientos con onda corta y ultrasonidos, sin incluir las exploraciones de ecografía. El motivo es la producción de calor local en el área del generador y del electrodo, que podría producir lesiones importantes en tejidos blandos, nervios y, lo que podría ser más grave, vasos sanguíneos.

La realización de resonancias magnéticas en pacientes portadores de equipos de estimulación vagal es posible, ya que si bien inicialmente se tenía cierta cautela ante esta prueba por la posibilidad de mover el electrodo o el generador, o por la producción de calor local, con posterioridad se ha comprobado que el único problema puede ser la desactivación del generador durante la aplicación del campo magnético, lo que resulta posible evitar mediante la colocación del generador en sentido paralelo al eje del cuerpo $^{49}$.

\section{Mecanismo de acción}

Aunque la forma de actuación de la estimulación intermitente vagal se desconoce, se piensa que podría inhibir la sincronización de los distintos núcleos que se deben descargar al unísono para que la actividad eléctrica cerebral normal se convierta en una actividad epileptógena. La estimulación cerebral retrógrada a través del nervio vago se planteó inicialmente como posibilidad por la amplia difusión de sus conexiones, por ser técnicamente asequible y por no llevar en su tronco fibras que producen dolor. El principal lugar donde el nervio proyecta sus aferencias es en el núcleo del tracto solitario. Otras fibras se proyectan 
a la formación reticular pontina, núcleo ambiguo, núcleo espinal del trigémino, área postrema, núcleo dorsal del vago y núcleo cunneatus. Desde estos núcleos intermedios, las aferencias continúan hacia el sistema Iímbico, hipotálamo, tálamo y corteza insular. El núcleo del tracto solitario además establece conexiones con otros núcleos del tronco de encéfalo, el locus coeruleus, los núcleos del rafe y la formación reticular. Estos núcleos, a su vez, pueden hacer variar directa o indirectamente la excitabilidad cortical y la actividad del sistema límbico. La estimulación puede alterar los circuitos en diferentes niveles, pero no se ha probado cuál de ellos justifica una acción antiepiléptica. Sin embargo, aún está por dilucidarse cómo la acción de estos circuitos influye sobre el control de las crisis epilépticas. El punto final parece ser un aumento de la hiperpolarización lenta en las neuronas corticales ${ }^{85}$. Un dato interesante es el aumento de la expresión del FOS, proteína nuclear indicativa de incremento de actividad neuronal, recogido por Naritoku et $\mathrm{al}^{54}$ en la amígdala posterior, el cíngulo, la corteza retroesplenial, los núcleos talámicos arcuato y ventromedial, los núcleos del vago, el locus coeruleus y los núcleos cocleares. En algunos ensayos en experimentación animal se ha demostrado además, que lesiones del locus coeruleus, tanto agudas como crónicas, disminuyen la eficacia de la estimulación vagal en el control de las crisis ${ }^{35}$.

Existen múltiples estudios que apoyan la eficacia de la estimulación eléctrica vagal en la epilepsia rebelde. Al aplicarla en diferentes animales de experimentación se ha observado su utilidad en varios de los tests habituales empleados para determinar el poder antiepiléptico y anticonvulsivante. Los trabajos clásicos de Bailey y Bremen ${ }^{5}$ $y$ de Zanchetti et $a^{86}$ demostraron cambios en el electroencefalograma (EEG), con aumento de ondas de frecuencias altas, aumento del umbral en el modelo de estricnina tópica y disminución de puntas intercríticas; sin embargo, estos datos no se aplicaron hasta que fueron confirmados más recientemente por otros investigadores, también con modelos de estricnina y pentiletilentrazol en perros y ratas y umbral de electroshock en ratones ${ }^{15,36.50,81,84}$. Rutecki ${ }^{65}$ en el modelo epiléptico de estricnina tópica en ratas, describe tanto sincronización como desincronización en distintas frecuencias en el EEG, y la disminución de puntas intercríticas. En gatos a los cuales se ha producido kindling eléctrico en la amígdala, la estimulación vagal retrasa o evita la producción de crisis espontáneas ${ }^{18}$. Produce, además, cambios en la actividad eléctrica normal, aumentando el sueño REM y la densidad de ondas PGO. También altera el comportamiento, produciendo aumento del apetito, bostezos y otras alteraciones como miosis, parpadeo, salivación, contracciones abdominales, desviación de la mirada hacia arriba y movimientos bruscos de la cabeza ${ }^{18}$. Algunos estudios refieren cambios en la frecuencia de base en el EEG, dependiendo de la intensidad de la estimulación. Utili- zando electrodos intracraneales se han encontrado cambios en la frecuencia de las descargas epileptiformes ${ }^{55}$. Otros estudios, sin embargo, no han confirmado cambios en el EEG, ni a simple vista ni en el análisis de frecuencias ${ }^{23,45,66}$. Incluso, para algunos autores, no evita la producción de nuevas crisis ${ }^{10}$.

Otra forma de estudiar los efectos de la estimulación vagal consiste en detectar los posibles cambios inducidos en el sistema nervioso central. Se han descrito la existencia de respuestas evocadas en la corteza cerebral, hipocampo, tálamo y cerebelo ${ }^{45}$. En los potenciales evocados se han observado resultados dispares en función de autores. Así, Naritoku et $\mathrm{al}^{53}$ han observado en tres pacientes, un aumento de latencia de la conducción periférica y central de los potenciales evocados somatosensoriales (PESS), sin cambios en los potenciales evocados visuales (PEV) o auditivos troncoencefálicos (PEA). Por el contrario, Hammond et $\mathrm{al}^{24}$ en un número mayor de pacientes y utilizando técnicas de estimulación y registro similares, no han encontrado cambios sobre los PESS, PEV o PEA después de la utilización de la estimulación eléctrica vagal de forma aguda o crónica.

Los efectos de la estimulación intermitente vagal sobre los diferentes estudios de imagen cerebrales funcionales en el humano, ofrecen una alternativa válida a los modelos experimentales de epilepsia para investigar sus mecanismos de acción. Así, la resonancia magnética con espectroscopia (RMS), la resonancia magnética funcional (fMRI), la tomografía por emisión de positrones (PET) y la tomografía computada por emisión simple de fotones (SPECT), permiten de forma no invasiva realizar un mapeo regional cerebral de flujo sanguíneo, metabolismo de glucosa, concentración de neurotransmisores, disponibilidad de neurorreceptores y otras funciones ${ }^{28}$. Los resultados objetivados con estas técnicas no son homogéneos. Estudios mediante PET con agua indicaron un aumento en el flujo sanguíneo cerebral durante las fases de estimulación del nervio vago izquierdo, siendo especialmente significativo en el tálamo derecho, corteza temporal posterior bilateral, putamen izquierdo y zona de hemisferio cerebeloso inferior izquierdo ${ }^{33,38}$ Henry et $\mathrm{al}^{26}$ encontraron un aumento de flujo en el hipotálamo, tálamo, corteza insular, giro poscentral, área inferior del cerebelo y cara rostral del bulbo, y una disminución de forma bilateral en hipocampo, amígdala y área posterior del giro singular. En un estudio posterior, estos mismos autores encontraron un aumento del flujo en ambos tálamos asociado a una reducción significativa del número de crisis, lo que sugeriría que el efecto antiepiléptico podría relacionarse con un aumento de actividad sinóptica en el tálamo ${ }^{27}$. Sin embargo, a pesar de estos hallazgos, existen estudios que recogen una disminución de la perfusión en diferentes zonas cerebrales tras la estimulación vagal ${ }^{94}$. De la misma forma, Vonck et $\mathrm{al}^{78}$, con estudios de SPECT con 
tecnecio-99 han destacado una hipoperfusión del tálamo homolateral, ya en las fases iniciales de la estimulación, sin encontrar correlación de estos hallazgos con cambios en la frecuencia de las crisis epilépticas. Presumiblemente, la fMRI podrá ser, en un futuro próximo, más utilizada para determinar cambios regionales en el flujo sanguíneo cerebral durante la estimulación vagal. Los únicos datos que se disponen en la actualidad a este respecto han sido obtenidos en pacientes con depresión sin crisis epilépticas tratados con estimulación intermitente vagal ${ }^{9}$.

La estimulación del nervio vago izquierdo es capaz de inducir cambios en la concentración de aminoácidos y fosfolípidos en el líquido cefalorraquídeo, pudiéndose inferir a través de los mismos una posible explicación de la acción antiepiléptica de la estimulación ${ }^{7,24}$. Ambos estudios muestran resultados similares. Así, los niveles de ácido gamaaminobutírico, estaban aumentados tanto en los pacientes con buena respuesta al tratamiento como en aquéllos que no se beneficiaron del mismo. La concentración de aminoácidos activadores glutamato y aspartato estaban descendidos en todos los grupos de pacientes, mientras que las del metabolito serotoninérgico 5-hidroxiindolacético estaban aumentados. Estos cambios no fueron estadísticamente significativos, pensando que reflejan más la acción de la estimulación vagal sobre la liberación de determinados neurotransmisores que una clara acción antiepiléptica. Por otro lado, el aumento de la concentración de fosfoetanolamina, un precursor de la membrana fosfolipídica, en los pacientes con buena respuesta clínica, sugeriría un incremento en el recambio de los componentes de la membrana neuronal, lo que podría indicar un efecto protector neuronal a través de la estabilización de su membrana, producido por la estimulación vagal.

Los estudios en animales no han servido únicamente para investigar el mecanismo de acción y valorar la actividad antiepiléptica, también han resultado útiles para determinar la máxima intensidad y parámetros de estimulación sin producir daño químico o eléctrico ${ }^{80}$. Esto es importante porque los parámetros eléctricos constituyen una variable fundamental relacionada con el control de las crisis y otros aspectos fisiológicos ${ }^{40}$.

\section{Resultados en el control de las crisis}

Los ensayos clínicos previos a la generalización de la técnica de estimulación intermitente vagal, se centraron en la eficacia, efectos secundarios y la comparación de resultados en función de la intensidad de corriente, alta o baja, del generador. Así, los primeros estudios demostraron una reducción de las crisis en la fase aguda del $28 \%$, con un $37 \%$ de pacientes que presentaban una disminución de las mismas superior al $50 \%{ }^{75}$. Con posterioridad, otros estudios en los que se incluían pacientes con epilepsias focales farmacorresistentes, evidenciaron a las 14 semanas de tratamiento, con estimulación de alta intensidad $(1.5 \mathrm{~mA}$, $30 \mathrm{~Hz}, 30 \mathrm{seg}$ cada $5 \mathrm{~min}$ ) una reducción de las crisis del $31 \%$; mientras que el grupo de pacientes con un estímulo inferior $(1.25 \mathrm{~mA}, 1 \mathrm{~Hz}, 30 \mathrm{seg}$ cada $90 \mathrm{~min})$ presentaba una disminución del $11 \%$. En el conjunto de pacientes incluidos en este estudio se obtuvo una reducción del $25 \%$ en el número de las crisis a los tres meses, y de un $32 \%$ en el seguimiento a lo largo de un año ${ }^{6}$. En estos casos, al dejar de funcionar el generador, la frecuencia de crisis se mantuvo estable durante las primeras semanas, volviendo después a la frecuencia habitual. Otros estudios hacen ya referencia a pacientes diagnosticados de epilepsia parcial o generalizada y con edades comprendidas entre los 13 y 60 años, obteniéndose igualmente una reducción de las crisis que por término medio fue del $46 \% \%^{25.39}$.

Los pacientes incluidos en los estudios controlados comentados anteriormente se los siguió de modo abierto a lo largo de periodos más prolongados de tiempo dando todos ellos unos valores similares de eficacia en el control de la crisis, con una reducción de crisis media de $35 \%$ en el primer año, $44.3 \%$ al cabo del segundo y $44.1 \%$ pasados tres años ${ }^{51}$.

En niños, la experiencia es menor pero los resultados son similares a los encontrados en los adultos ${ }^{16,37,79}$. Hornig et $\mathrm{al}^{29}$, en un estudio sobre 19 niños, obtuvieron una mejoría de la frecuencia de crisis de un $50 \%$ en diez de ellos, y en seis la reducción fue superior al $90 \%$. Otros autores enfatizan sobre los buenos resultados en la reducción de las crisis, que en algunos casos llegan a más del $90 \%$ en niños con síndrome de Lennox-Gastaut ${ }^{30,47}$. Recientemente, datos de un grupo de pacientes con edades comprendidas entre los 11 meses y 16 años indican una eficacia y seguridad similares a las de los adultos, ampliando el espectro de las crisis que mejoran a las crisis tónicas y a las ausencias ${ }^{57}$.

Durante los últimos años se empiezan a recoger publicaciones en las que la eficacia de la estimulación intermitente vagal no se limita exclusivamente a pacientes con epilepsia parcial sino que se han recogido resultados similares a los anteriormente expuestos en pacientes con epilepsia generalizada idiopática, síndrome de Lennox-Gastaut ${ }^{8}$, crisis parciales complejas o secundariamente generalizadas ${ }^{2}$, epilepsia bitemporal y epilepsia secundariamente generalizada o parcial ${ }^{52,61}$. En adultos de edad avanzada, los resultados también son prometedores ${ }^{69}$.

Aunque no con tanta frecuencia, existen grupos cuya experiencia con la estimulación vagal no es tan positiva. Así, en algunas series aparecen reducciones de la frecuencia de crisis de tan sólo el $17 \%{ }^{56} \mathrm{e}$ incluso un marcado empeoramiento de las crisis y aparición de crisis de nuevos tipos ${ }^{34}$.

\section{Otras acciones de la estimulación vagal}

Además de la eficacia sobre la reducción de las crisis se 
han aportado múltiples datos sobre otros indicadores clínicos. Pacientes y familiares han confirmado un beneficio en otras áreas como el nivel de alerta, la memoria, el estado de ánimo y la expresión verbal. La tranquilidad que confiere el hecho de poder detener la crisis es un factor muy apreciado. La reducción de la dosis y el número de los fármacos antiepilépticos contribuye también a la mejoría global, a la disminución de los posibles efectos secundarios y a mejorar el estado de alerta ${ }^{73}$. En este sentido, en un paciente epiléptico con síndrome de Unverricht-Lundborg mejoró no sólo el control de la crisis, sino también la sintomatología cerebelosa ${ }^{70}$.

\section{Efectos secundarios y complicaciones}

La técnica de estimulación eléctrica vagal puede tener complicaciones derivadas del procedimiento quirúrgico o bien de la presencia de un cuerpo extraño. Otras provienen de la actividad propia del sistema, debido a la excitación eléctrica inducida en el nervio vago o en territorios vecinos.

La intervención quirúrgica para la implantación del sistema se considera como una cirugía de bajo riesgo, aunque pueden ocurrir hemorragias o infecciones, de modo poco frecuente como en otras cirugías del área cervical. Fisher y Handforth ${ }^{19}$ recogen un caso de parálisis de la cuerda vocal izquierda que persistía tras un año de seguimiento.

Los efectos secundarios más frecuentes son ronquera, tos, dolor cervical, disfonía y alteración del tono de voz que suelen mantenerse en los periodos de estimulación. La disfonía y la sensación anormal en la faringe son más frecuentes y molestas si la estimulación se lleva a cabo con parámetros de estimulación alta. La disnea y el hipo aparecen con mucha menor frecuencia, pudiendo producir ocasionalmente también cefalea o náuseas. En niños con retraso psicomotor se han descrito algunos casos de dificultad para la deglución ${ }^{47}$.

Entre los nervios vecinos en los que se han recogido diferentes alteraciones están el plexo simpático cervical ${ }^{32}$, el nervio frénico ${ }^{42}$ y el glosofaríngeo ${ }^{12}$, además de la presentación de algún caso de tortícolis debido a la inducción de un espasmo en el músculo esternocleidomastoideo por afectación del nervio espinal ${ }^{31}$.

Una de las complicaciones más graves es la alteración del ritmo cardíaco. La implantación del electrodo en el nervio vago izquierdo intenta evitar estas alteraciones pero, a pesar de ello, ha habido casos de bradicardia e incluso asistolia ${ }^{4,41,43}$. Tatum et $\mathrm{al}^{72}$ refieren haber tenido cuatro casos de asistolia en el propio quirófano durante la estimulación, por ello, recomiendan que las pruebas posteriores se realicen con control continuo electrocardiográfico. Otros trabajos publicados inciden en la ausencia de cambios en la frecuencia cardíaca con la estimulación ${ }^{68}$.
Los pacientes con enfermedad pulmonar obstructiva crónica o con apneas del sueño deben controlarse más detenidamente por la posibilidad de empeorar la función respiratoria o incrementar el índice de apneas ${ }^{46,48}$. También se ha descrito una mayor frecuencia de muerte súbita no explicada en pacientes epilépticos tratados con estimulación vagal. La explicación de este hecho reside probablemente en la selección de los pacientes, ya que se trata de pacientes epilépticos farmacorresistentes, con mayor frecuencia basal de crisis y, en consecuencia, mayor riesgo general de muerte súbita ${ }^{3}$.

Los problemas técnicos suelen ser poco frecuentes. En el caso de objetivarse un aumento de resistencia del electrodo, la consecuencia es un mayor gasto de batería. En algún caso aislado se ha presentado un cortocircuito en el sistema que produjo una estimulación directa y problemas en la programación del programador ${ }^{62}$.

Globalmente se podría concluir que los efectos secundarios y complicaciones de la estimulación eléctrica vagal son poco frecuentes e importantes, y suelen disminuir o desaparecer al ajustar correctamente los parámetros de estimulación.

\section{Conclusiones. Aproximación crítica a la técnica}

La estimulación eléctrica intermitente vagal es un tipo de terapéutica antiepiléptica relativamente nueva, que tradicionalmente ha sido utilizada en pacientes en los que han fallado los ensayos clínicos con diferentes fármacos. Los datos de los diferentes estudios clínicos indican que estos enfermos han sido tratados por término medio con ocho o nueve fármacos antiepilépticos diferentes, de forma aislada o combinada, antes de tratarse con neuroestimulación. Igualmente, la historia media de epilepsia en los pacientes de todos estos estudios era de aproximadamente veinte años. Es por ello que se hace necesaria la utilización de esta técnica en epilepsias refractarias de forma más precoz, en fases más tempranas de diagnóstico y cuando se han empleado muchas menos drogas antiepilépticas.

Varios factores necesitan ser evaluados en las estrategias a largo plazo del tratamiento de los pacientes epilépticos. La reducción en el número e intensidad de las crisis, la calidad de vida y los efectos secundarios y complicaciones son factores claves para analizar los resultados de los diferentes tratamientos. Muchos de los fármacos anticomiciales tienen importantes efectos secundarios sobre el sistema nervioso central como la sedación, disminución de la cognición, atención y concentración, pérdida de memoria, alteraciones en la coordinación motora y trastornos conductuales y caracterológicos entre otros. Además este tipo de productos en mono o politerapia tienen una toxicidad sistémica expresada en forma de leucopenia, reducción del fibrinógeno, alteraciones hepáticas inespecíficas, hirsutismo, ganancia 
de peso, erupciones y alergias cutáneas, y gingivitis, que con frecuencia resultan en serias complicaciones generales. Por el contrario, la estimulación del nervio vago, si bien no está exenta de efectos secundarios, suelen reducirse a ronquera, tos, dolor cervical, disfonía y alteración en el timbre de voz, considerados moderados y transitorios, al limitarse en general al periodo de estimulación. Suelen minimizarse ajustando correctamente los parámetros de la misma. No se han recogido durante la estimulación alteraciones en la atención y concentración, en la conciencia, en la visión, en la coordinación o en el carácter.

Los resultados de los ensayos clínicos y la experiencia acumulada en los últimos años, tal como se ha comentado en anteriores apartados, sitúan a la estimulación vagal como una posibilidad más dentro del arsenal terapéutico de la epilepsia. Aunque es un sistema económicamente gravoso, la disminución de las crisis y del status epiléptico hace bajar significativamente los cuidados médicos. Por eso, aunque estos pacientes deben continuar con fármacos antiepilépticos, la relación coste/beneficio parece positiva. Sin embargo, a pesar de los buenos resultados obtenidos en general, existen grandes controversias a la hora de aplicar este tipo de técnica, al no saber con exactitud su eficacia en cada uno de los diversos tipos de crisis y síndromes epilépticos. Se hace imprescindible conocer los factores pronósticos que posibiliten la mejor respuesta clínica en cada tipo de epilepsia, puesto que en muchos casos es más importante el control de una crisis específica que la reducción en la frecuencia de la misma.

En esta línea, existen una serie de problemas prácticos que han impedido una globalización mayor de la estimulación intermitente vagal. Así, todavía no se dispone de una respuesta fisiológica medible que permita individualizar la estimulación. De igual forma, no se conoce, de una manera prospectiva, qué tipo de epilepsia o paciente epiléptico es un candidato ideal a ofrecer una respuesta positiva a la estimulación. Finalmente, se puede decir, que en la actualidad parece elevado el coste inicial del equipo y aparato de estimulación.

Se hace así necesario el continuar investigando y ensayando este tipo de técnica en grupos más amplios de pacientes para definitivamente, conocer con exactitud su eficacia y seguridad y poder determinar quiénes son los candidatos epilépticos idóneos para recibir este tratamiento. Por otro lado, estos nuevos estudios deberán indicar los parámetros de estimulación eléctrica más óptimos que produzcan el máximo beneficio con menos efectos secundarios. Finalmente, se precisan también de estudios tecnológicos de ingeniería biomédica que permitan perfeccionar los aspectos técnicos del equipo de estimulación y abaraten los costes de producción y venta del sistema, porque sólo así se podrá generalizar y tendrán acceso a esta moderna técnica un mayor número de pacientes de todo el mundo.
El mecanismo de acción, es otro punto conflictivo dentro de la estimulación vagal, sometido a muchas hipótesis y controversias. Así, los cambios electroneurofisiológicos recogidos tras la estimulación, tanto en el campo clínico como experimental, no son nada concluyentes. Un grupo más numeroso de autores recogen en los diferentes registros un aumento de ondas de alta frecuencia, una disminución de las puntas intercríticas, una sincronización-desincronización a diferentes frecuencias en el EEG, cambios en la frecuencia base del EEG y cambios en la frecuencia de descargas epileptiformes, todo ello expresión de un claro beneficio de la estimulación. Otros investigadores por el contrario no recogen ningún tipo de cambios en el registro del EEG ni en el análisis de frecuencias. Igualmente, existen controversias a la hora de analizar los potenciales evocados somatosensoriales tras la estimulación, pudiendo aumentar o disminuir en función de estudios. Existe por el contrario, un acuerdo general acerca de la ausencia de registros positivos en los potenciales evocados auditivos o visuales en estos enfermos.

Los resultados recogidos en las diferentes técnicas de imagen funcional (RMS, fMRI, PET, SPECT) en los pacientes tratados con estimulación vagal son, además de escasos poco homogéneos, recogiéndose tanto aumentos como disminución de la perfusión sanguínea en las diferentes localizaciones cerebrales estudiadas.

Con respecto a la determinación de neurotransmisores y otros metabolitos en líquido cefalorraquídeo también son pocos los estudios que se han realizado, mostrando en general un incremento de moduladores inhibidores y un descenso de metabolitos activadores de la transmisión sináptica tras la estimulación. En estos casos, además, se ha recogido un incremento de productos metabólicos de recambio de membrana neuronal que indicaría una mayor regeneración de la misma en estos pacientes.

Finalmente, se echan de menos estudios experimentales, tanto en animales de experimentación sanos como en modelos epilépticos, que incidan sobre los cambios de perfusión cerebral tras la estimulación eléctrica o la determinación del daño químico o eléctrico, tisular local o cerebral que se podría producir tras el empleo crónico de la misma.

\section{Bibliografía}

1. Alsaadi, T.M., Laxer, K.D., Barbaro, N.M., Marks, W.J., García, P.A.: Vagus nerve stimulation for the treatment of bilateral independent temporal lobe epilepsy. Epilepsia 2001; 42: 954-956.

2. Amar, A.P., Heck, C.N., Levy, M.L.,et al.: An institutional experience with cervical vagus nerve trunk stimulation for medically refractory epilepsy: rationale, technique, and outcome. Neurosurgery 1998; 43: 1265-1276.

3. Annegers, J.F., Coan, S.P.; Hauser, W.A., Leestma, J., 
Duffell, W., Tarver, B.: Epilepsy, vagal nerve stimulation by the NCP system, mortality, and sudden, unexpected, unexplained death. Epilepsia 1998; 39: 206-212.

4. Asconapé, J., Moore, D., Zipes D., Hartman, L., Duffell, W.H.: Bradycardia and asystole with the use of vagus nerve stimulation for the treatment of epilepsy: a rare complication of intraoperative device testing. Epilepsia 1999; 40: 14521454.

5. Bailey, P., Bremer, F.: A sensory cortical representation of the vagus nerve with a note on the effects of low blood pressure on the cortical electrogram. J Neurophysiol 1938; 1 : 405-412.

6. Ben-Menachem, E, Manon-Espaillat, R., Ristanovic, R. et al: First International Vagus Nerve Stimulation Study Group: Vagus nerve stimulation for the treatment of partial seizures: 1-A controlled study of effect on seizures. Epilepsia 1994; 35: 616-626.

7. Ben-Menachem, E., Hamberger, A., Hedner, T., et al: Effects of vagus nerve stimulation on amino acids and other metabolites in the CSF of patients with partial seizures. Epilepsy Res 1995; 20: 221-227.

8. Ben-Menachem, E., Hellstrom, K., Waldton, C., Augustinsson, L.E.: Evalua-tion of refractory epilepsy treated with vagus nerve stimulation for up 5 years. Neurology 1999; 52: 1265-1276.

9. Bohning, D.E., Lomarev, M.P., Denslow, S., Nahas, Z., Shastri, A., George, M.S.: Feasibility of vagus nerve stimulation-synchronized blood oxygenation level-dependent functional MRI. Invest Radio! 2001; 36: 470-479.

10. Dasheiff, R.M., Sandberg, T., Thompson, J., Arrambide, S.: Vagal nerve stimulation does not unkindle seizures. J Clin Neurophysiol 2001; 18: 68-74.

11. Devinsky, O., Pacia, S.: Epilepsy surgery. Neurol Clin N Am 1993; 11: 951-971.

12. Duhaime, A., Relamed, M., Clancy, R.: Tonsillar pain mimicking glosso-pharyngeal neuralgia as a complication of vagus nerve stimulation: case report. Epilepsia 2000; 41: 903905.

13. Engel, J.: Surgery for seizures. N Engl J Med 1996; 334: 647-652.

14. Espinosa, J., Aiello, M.T., Naritoku, D.K.: Surg Neurol Revision and removal of stimulating electrodes following long-term therapy with the vagus nerve stimulator. Surg Neurol 1999; 51: 659-664.

15. Fanselow, E.E., Reid, A.P., Nicolelis, M.A.: Reduction of pentylenetetrazole induced seizure activity in awake rats by seizure-triggered trigeminal nerve stimulation. J Neurosci 2000; 20: 8160-8168.

16. Farooqui, S., Boswell, W., Hemphill, J.M., Pearlman, E.: Vagus nerve stimulation in pediatric patients with intractable epilepsy: case series and operative technique. Am Surg 2001; 67: 119-121.

17. Feinstein, B., Gleason, C.A., Libet, B.: Stimulation of locus coeruleus in man. Preliminary trials for spasticity and epilepsy. Stereotact Funct Neurosurg 1989; 52: 26-41.

18. Fernández, GA., Martínez, A., Valdés, C.A., Magdaleno, M.V., Martínez, D., Fernández, M.R.: Vagus nerve prolonged stimulation in cats: effects on epileptogenesis (amygdala electrical kindling), behavoriai and electrographic changes. Epilepsia 1999; 40: 822-829.

19. Fisher, R.S., Handforth, A.: Reassessment of vagus nerve stimulation for epilepsy: a report of the Therapeutics and Technology Assessment Subcommittee of the American Academy of Neurology. Neurology 1999; 53: 666-669.

20. Fisher, R.S., Mirski, M., Krauss, G.L.: Brain stimulation. En Engel J Jr y Pedley TA (eds). Epilepsy: A Comprehensive Textbook. Philadelphia; Lippincott-Raven, 1998; pp.1867-8875.

21. Fisher, R.S., Uematsu, S., Krauss, G.L., Cysyk, B.J., McPherson, R., Lesser, R.P.: Placebo-controlled pilot study of centromedian thalamic stimulation in treatment of intractable seizures. Epilepsia 1992; 33: 841-851.

22. Gowers, W.R.: Epilepsy and other chronic convulsive diseases: their causes, symptoms, and treatment. London; JA Churchill, 1881; 41, pp. 93-97, pp. 287-290.

23. Hammond, E.J., Uthman, B.M., Reid, SA., Wilder, B.J., Ramsay, R.E.: Vagus nerve stimulation in humans: neurophysiological studies and electro-physiological monitoring. Epilepsia 1990; 31 suppl 2: S51-S59.

24. Hammond, E.J., Uthman, B.M., Reid, S.A., Wilder, B.J.: Electrophysiologic studies of cervical vagus nerve stimulation in humans. II. Evoked potentials. Epilepsia 1992; 33: 1021-1028.

25. Handforth, A., DeGiorgio, C.M., Schachter, S.C., Uthman, B.M., Naritoku, D.K., Tecoma, E.S.: Vagus nerve stimulation therapy for partial-onset seizures: a randomized active-control trial. Neurology 1998; 51: 48-55.

26. Henry, T.R., Bakay, R.A., Votaw, J.R., Pennell, P.B., Epstein, C.M., Faber, T.L.: Brain blood flow alterations induced by therapeutic vagus nerve stimulation in partial epilepsy. I. Acute effects at high and low levels pf stimulation. Epilepsia 1998; 39: 983-990.

27. Henry, T.R., Votaw, J.R., Pennell, P.B., Epstein, C.M., Bakay, R.A., Faber, T.L.: Acute blood flow changes and efficacy of vagus nerve stimulation in partial epilepsy. Neurology 1999; 52: 1166-1173.

28. Henry, T.R., Duncan, J.S., Berkovic, S.F.: Functional Imaging in the Epilepsies. Philadelphia; Lippincott Williams y Wilkins, 2000.

29. Hornig, G.W., Murphy, J.V., Schallert, G., Tilton, C.: Left vagus nerve stimul-ation in children with refractory epilepsy: an update. South Med J 1997; 90: 484-488.

30. Hosain, S., Nikalov, B., Harden, C., Li, M., Fraser, R., Labar, D.: Vagus nerve stimulation treatment for Lennox-Gastaut syndrome. J Child Neurol 2000; 15: 509-512.

31. Iriarte, J., Lázaro, D., Viteri, J., Artieda, J.: Tetaniza- 
ción del esternocleido-mastoideo inducido por estimulador vagal. Neurología 2000; 15: 556.

32. Kim, W., Clancy, R.R., Liu, G.T.: Homer syndrome associated with implantation of a vagus nerve stimulator. Am J Ophthalmol 2001; 131: 383-384.

33. Ko, D., Heck, C., Grafton, S., Apuzzo, M.L., Couldwell, W.T., Chen, T.: Vagus nerve stimulation activates central nervous system structures in epileptic patients during PET H2(15)O blood flow imaging. Neurosurgery 1996; 39: 426430 .

34. Koutroumanidis, M., Hennessy, M.J., Binnie, C.D., Polkey, C.E.: Aggravation of partial epilepsy and emergence of new seizure type during treatment with VNS. Neurology 2000; 55: 892-893.

35. Krahl, S.E., Clark, K.B., Smith, D.C., Browning, R.A.: Locus coeruleus lesions suppress the seizure-attenuating effects of vagus nerve stimulation. Epilepsia 1998; 39: 709714.

36. Krahl, S.E., Senanayake, S.S., Handforth, A.: Seizure suppression by systemic epinephrine is mediated by the vagus nerve. Epilepsy Res 2000; 38: 171-175.

37. Labar, D.: Vagus nerve stimulation for intractable epilepsy in children. Dev Med Child Neurol 2000; 42: 496-499.

38. Labar, D., Nikolov, B., Tarver, B., Fraser, R.: Vagus nerve stimulation for symptomatic generalizad epilepsy: a pilot study. Epilepsia 1998; 39: 201-205.

39. Labar, D., Murphy, J., Tecoma, E.: Vagus nerve stimulation for medicat-ionresistant generalized epilepsy. E04 VNS Study Group. Neurology 1999; 52: 1510-1512.

40. Landy, H.J., Ramsay, R.E., Slater, J., Casiano, R.R., Morgan, R.: Vagus nerve stimulation for complex partial seizures: surgical technique, safety, and efficacy. J Neurosurg 1993; 78: 26-31.

41. Lanska, D.J.: Ventricular asystole during vagus nerve stimulation for epilepsy in humans. Neurology 2000; 54: 775.

42. Leijten, F.S., Van-Rijen, P.C.: Stimulation of the phrenic nerve as a complication of vagus nerve pacing in a patient with epilepsy. Neurology 1998; 51: 1224-1225.

43. Lesser, R.P.: Ventricular asystole during vagus nerve stimulation for epilepsy in humans. Neurology 2000; 54: 776.

44. Lockard, J.S., Congdon, W.C.: Effects of vagal stimulation on seizure rate in monkey model. Epilepsia 1986; 27 : 626.

45. Lockard, J.S., Congdon, W.C., DuCharme, L.L.: Feasibility and safety of vagal stimulation in monkey model. Epilepsia 1990; 31 suppl 2: S20-S26.

46. Lotvall, J., Lunde, H., Augustinsson, L.E., Hedner, T., Svedmyr, N., Ben-Menachem, E.: Airway effects of direct left-sided cervical vagal stimul-ation in patients with complex partial seizures. Epilepsy Res 1994; 18: 149-154.

47. Lundgren, J., Amark, P., Blennow, G., Stromblad, L.G., Wallstedt, L.: Vagus nerve stimulation in 16 children with refractory epilepsy. Epilepsia 1998; 39: 809-813.
48. Malow, B.A., Edwards, J., Marzec, M., Sagher, O., Fromes, G.: Effects of vagus nerve stimulation on respiration during sleep: a pilot study. Neurology 2000; 55: 1450-1454.

49. Maniker, A., Liu, W.C., Marks, D., Moser, K., Kalnin, A.: Positioning of vagal nerve stimulators: technical note. Surg Neurol 2000; 53: 178-181.

50. McLachlan, R.S.: Suppression of interictal spikes and seizures by stimulation of the vagus nerve. Epilepsia 1993; 34 : 918-923.

51. Moms, G.L., Mueller, W.M.: Long-term treatment with vagus nerve stimulation in patients with refractory epilepsy. The Vagus Nerve Stimulation Study Group E01-E05. Neurology 1999; 53: 1731-1735.

52. Morrow, J.I., Bingham, E., Craig, J.J., Gray, W.J.: Vagal nerve stimulation in patients with refractory epilepsy. Effect on seizure frequency, severity and quality of life. Seizure 2000; 9: 442-445.

53. Naritoku, D.K., Morales, A., Pencek, T.L., Winkler, D.: Chronic vgaus nerve stimulation increases the latency of the thalamocortical somatosensory evoked potential. Pacing Clin Electrophysiol 1992; 15: 1572-1578.

54. Naritoku, D.K., Terry, W.J., Helfert, R.H.: Regional induction of fos immunoreactivity in the brain by anticonvulsant stimulation of the vagus nerve. Epilepsy Res 1995; 22: 53-62.

55. Olejniczak, P.W., Fisch, B.J., Carey, M., Butterbaugh, G., Happel, L., Tardo, C.: The effect of vagus nerve stimulation on epileptiform activity recorded from hippocampal depth electrodes. Epilepsia 2001; 42: 423-429.

56. Parker, A.P., Polkey, C.E., Binnie, C.D., Madigan, C., Ferrie, C.D., Robinson, R.O.: Vagal nerve stimulation in epileptic encephalopathies. Pediatrics 1999; 103: 778-782.

57. Patwardhan, R.V., Stong, B., Bebin, E.M., Mathisen, J., Grabb, P.A.: Efficacy of vagal nerve stimulation in children with medically refractory epilepsy. Neurosurgery 2000; 47: 1353-1357.

58. Penry, J.K., Dean, J.C.: Prevention of intractable partial seizures by intermittent vagal stimulation in humans: Preliminary results. Epilepsia 1990; 31 suppl 2: S40-S43.

59. Pompeiano, O., Swett, J.E.: EEG and behavioral manifestations of sep induced by cutaneous nerve stimulation in normal cats. Arch Ital Biol 1962; 100: 311-342.

60. Porter, R.J.: Epilepsy: Prevalence, classification, diagnosis, and prognosis. En Apuzzo MLJ (ed). Neurosurgical Aspects of Epilepsy. Park Ridge; AANS, 1991; pp 17-26.

61. Rafael, H., Moromizato, P.: Vagus nerve stimulation (VNS) may be use-ful in treating patients with symptomatic generalized epilepsy. Epilepsia 1998; 39: 1018.

62. Ramsay, R.E., Uthman, B.M., Augustinsson, L.E., Upton, A.R., Naritoku, D., Willis, J.: Vagus nerve stimulation for treatment of partial seizures. 2. Safety, side effects, and tolerability. First International Vagus Nerve Stimulation Study Group. Epilepsia 1994; 35: 627-636. 
63. Reid, S.A.: Surgical technique for implantation of the neurocybernetic prosthesis. Epilepsia 1990; 31 suppl 2: S38S39.

64. Ring, H.A., White, S., Costa, D.C., Pottinger, R., Dick, J.P., Koeze, T.: SPECT study of the effect of vagal nerve stimulation on thalamic activity in patients with epilepsy. Seizure 2000; 9: 380-384.

65. Rutecki, P.: Anatomical, physiological, and theoretical basis for the antiepileptic effect of vagus nerve stimulation. Epilepsia 1990; 31 suppl 2: S1-S6.

66. Salinsky, M.C., Burchiel, K.J.: Vagus nerve stimulation has no effect on awake EEG rhythms in humans. Epilepsia 1993; 34: 299-304.

67. Schweitzer, A., Wright, S.: Effects on the knee jerk of stimulation of the central end of the vagus and of various changes in circulation and respiration. J Physiol 1937; 88: 459-475.

68. Setty, A.B., Vaughn, B.V., Quint, S.R., Robertson, K.R., Messenheimer, J.A.: Heart period variability during vagal nerve stimulation. Seizure 1998; 7: 213- 217.

69. Sirven, J.I., Sperling, M., Naritoku, D., Schachter, S., Labar, D., Holmes, M.: Vagus nerve stimulation therapy for epilepsy in older adults. Neurology 2000; 54: 1179-1182.

70. Smith, B., Shatz, R., Elisevich, K., Bespalova, I.N., Burmeister, M.: Effects of vagus nerve stimulation on progressive myoclonus epilepsy of Unverri-cht-Lundborg type. Epilepsia 2000; 41: 1046-1048.

71. Spencer, D.D., Spencer, S.S.: Surgery for epilepsy. Neurol Clin 1985; 3: 313-330.

72. Tatum, W.O., Moore, D.B., Stecker, M.M., Baltuch, G.H., French, J.A., Ferreira, J.A.: Ventricular asystole during vagus nerve stimulation for epilepsy in humans. Neurology 1999; 52: 1267-1269.

73. Tatum, W.O., Johnson, K.D., Goff, S., Ferreira, J.A., Vale, F.L.: Vagus nerve stimulation and drug reduction. Neurology 2001; 56: 561-563.

74. Uthman, B.M., Wilder, B.J., Hammond, E.J., Reid, S.A.: Efficacy and safety of vagus nerve stimulation in patients with complex partial seizures. Epilepsia 1990; 31 suppl 2: S44-S50.

75. Uthman, B.M., Wilder, B.J., Penry, J.K., Dean, C., Ramsay, R.E., Reid, SA.: Treatment of epilepsy by stimulation of the vagus nerve. Neurology 1993; 43: 1338-1345.

76. Vagus Nerve Stimulation Study Group: A randomized controlled trial of chronic vagus nerve stimulation for treat- ment of medically intractable seizures. Neurology 1995; 45: 224-230.

77. Velasco, F., Velasco, M., Velasco, A.L., Jiménez, F., Márquez, I., Rise, M.: Electric stimulation of the centromedian thalamic nucleus in control of seizures: long-term studies. Epilepsia 1995; 36: 63-71.

78. Vonck, K., Boon, P., Van-Laere, K., D'Have, M., Vandekerckhove, T., O'Connor, S.: Acute single photon emission computed tomographic study of vagus nerve stimulation in refractory epilepsy. Epilepsia 2000; 41: 601-609.

79. Wakai, S., Kotagal, P.: Vagus nerve stimulation for children and adoles-cents with intractable epilepsies. Pediatr Int 2001; 43: 61-65.

80. Woodbury, D.M., Woodbury, J.W.: Effects of vagal stimulation on experimentally induced seizures in rats. Epilepsia 1990; 31 suppl 2: S7-S19.

81. Woodbury, J.W., Woodbury, D.M.: Vagal stimulation reduces the severity of maximal electroshock seizures in intact rats: use of cuff electrode for stimulating and recording. Pacing Clin Electrophysiol 1991; 14: 94-107.

82. Wright, G.D., McLellan, D.L., Brice, J.G.: A doubleblind trial of chronic cerebellar stimulation in twelve patients with severe epilepsy. J Neurol Neurosurg Psychiatry 1984; 47 : 769-774.

83. Zabara, J.: Time course of seizure control to brief, repetitive stimuli. Epilepsia 1985; 26: 518.

84. Zabara, J.: Inhibition of experimental seizures in canines by repetitive vagal stimulation. Epilepsia 1992; 33: 10051012 .

85. Zagon, A., Kemeny, AA.: Slow hyperpolarization in cortical neurons: a possible mechanism behind vagus nerve stimulation therapy for refractory epilepsy?. Epilepsia 2000; 41: 1382-1389.

86. Zanchetti, A., Wang, S.C., Moruzzi, G.: The effect of vagal afferent stimulation on the EEG pattern of the cat. Electroencephalogr Clin Neurophysiol 1952; 4: 357-361.

García-March, G ; Sánchez-Ledesma, M.J.; Broseta, J.: Estimulación eléctrica vagal en el tratamiento de la epilepsia rebelde. Situación actual. Neurocirugía 2008; 19: 416-426.

Correspondencia postal: Guillermo García-March. Servicio de Neurocirugía. Hospital Clínico Universitario. Avda.Vicente Blasco Ibáñez, 17. 46010-Valencia. 